This item was submitted to Loughborough's Research Repository by the author.

Items in Figshare are protected by copyright, with all rights reserved, unless otherwise indicated.

\title{
Influence of different fully non-stationary artificial time histories generation methods on the seismic response of frequency-dependent structures
}

\section{PLEASE CITE THE PUBLISHED VERSION}

https://2021.uncecomp.org/

\section{PUBLISHER}

Eccomas Proceedia

VERSION

AM (Accepted Manuscript)

\section{PUBLISHER STATEMENT}

This paper was accepted for publication in the UNCECOMP 2021 Proceedings and the definitive published version is available at https://www.eccomasproceedia.org/conferences/thematic-conferences/uncecomp-2021.

LICENCE

CC BY-NC-ND 4.0

\section{REPOSITORY RECORD}

Genovese, Federica, Giuseppe Muscolino, and Alessandro Palmeri. 2021. "Influence of Different Fully Nonstationary Artificial Time Histories Generation Methods on the Seismic Response of Frequency-dependent Structures". Loughborough University. https://hdl.handle.net/2134/14575203.v1. 


\title{
INFLUENCE OF DIFFERENT FULLY NON-STATIONARY ARTIFICIAL TIME HISTORIES GENERATION METHODS ON THE SEISMIC RESPONSE OF FREQUENCY-DEPENDENT STRUCTURES
}

\author{
Federica Genovese $^{1,2^{*}}$, Giuseppe Muscolino $^{2}$ and Alessandro Palmeri ${ }^{1}$ \\ ${ }^{1}$ School of Architecture, Building and Civil Engineering, Loughborough University, \\ Sir Frank Gibb, Loughborough LE11 3TU, England, United Kingdom \\ F.Genovese@Lboro.ac.uk, A.Palmeri@Lboro.ac.uk \\ ${ }^{2}$ Department of Engineering, University of Messina, \\ C.da di Dio, Villaggio S. Agata, 98166, Messina, Italy \\ fedgenovese@unime.it,gmuscolino@unime.it
}

\begin{abstract}
Advances in computational power and numerical optimization routines enable the application of rigorous simulation and optimization techniques for the design and assessment of structural and geotechnical $(S \& G)$ systems. Nowadays, the state of practice in the seismic design of high-importance structures has progressively moved toward the use of time-history dynamic analysis that are highly sensitive to the way of modelling the dynamic action. To be reliable, this type of analysis requires efficient methodologies for the selection of the seismic inputs used as ground excitations.

In this paper, a new stochastic approach, based on the use of the wavelet transform is proposed to generate an arbitrary number of seismic records having the same characteristics of a target accelerogram. The choice of the amplitude and number of bands in which to partition the frequency domain is a key source of variability to consider for the generation of samples with the desired time-varying amplitude and frequency content.

To evaluate the influence of the use of an alternative fully non-stationary artificial accelerograms generation methods, a comparison between the proposed method and the one recently proposed by the first two authors is also presented in this paper. Specifically, in order to quantify the influence of accelerograms models on the seismic response, a structural system with viscoelastic damping is analysed, representative of a broad range of frequencydependent $S \& G$ assets.
\end{abstract}

Keywords: Fully non-stationary models, Harmonic Wavelet Transforms, Artificial accelerograms, Time-history dynamic analyses, Maxwell Model. 


\section{INTRODUCTION}

The pseudo-acceleration response spectrum, typically used by international seismic codes for modelling the earthquake-induced ground shaking, significantly facilitates the design of regular structure through proper modal combination rules. However, there are many cases (e.g. seismic site response analysis and seismic design of structures with energy dissipation devices) in which the use of the elastic design response spectrum is not considered appropriate and non-linear dynamic time-history analyses are preferred. In these situations, the use of a suite of real accelerograms is an attractive option for modelling the seismic excitation; thus, different Ground Motion Selection and Modification methods have been proposed in literature (see e.g. [1-4]).

As evidenced in [5-7], the result of the selection procedures is influenced by multiple sources of uncertainties related to the seismic hazard at the site of interest and to the mechanical characteristics of the soil layers. As a consequence, it could be very difficult, if not impossible, to select an adequate number of recorded accelerograms without applying large scale factors to each record of the set, which in turn would distort the original signals characteristics.

A suitable alternative for the definition of the input ground motion consists in the use of sets of artificial accelerograms, generated by procedures able to capture the large variability of the seismological parameters observed in recorded time-histories (see e.g [8,9]).

The wavelet transform represents a useful tool to perform a joint-time frequency representation of non-stationary signals; for this reason, different wavelet-based approaches have been used for simulating artificial non-stationary accelerograms (see e.g. [10,11]).

In this paper, a new stochastic artificial accelerograms generation method, based on the use of circular wavelets transform $(C W T)$, is presented.

In comparison to other generation strategies available in the literature, the proposed procedure enables the generation of artificial time histories without the need of defying the evolutionary power spectral density (EPSD) function of the ground acceleration.

The correct choice of the amplitude and number of bands in which to partition the frequency domain is one of the main sources of variability to consider to achieve the generation of samples with the desired non-stationary characteristics.

In order to quantify the influence of different accelerograms models on the seismic response, a comparison between the proposed method and the piecewise EPSD function method, recently proposed in [12] is also reported in this paper.

The second stochastic generation method, for a given target accelerogram, requires the following steps: $i$ ) find a fully non-stationary model of earthquake ground motion such that the target accelerogram may be considered as one of its samples; ii) evaluate the mean elastic response spectrum of a set of generated fully non-stationary accelerogram samples; iii) satisfy the compatibility with the elastic target response spectrum by means of an iterative procedure.

In order to highlights the performance of these two alternative probabilistic models on the seismic response, a structural system with frequency-dependent damping, based on the viscoelastic Maxwell model, is analysed in this paper.

\section{WAVELET-BASED FULLY NON-STATIONARY GENERATION METHOD}

The wavelet analysis consists in the expansion of a given signal in terms of "wavelets", which are generated by scaling and shifting a chosen function called "mother wavelet". Among all different types of wavelets, the "harmonic" and "musical" ones proposed in [13] are particularly useful for dynamic analysis. These families of wavelets are complex-valued functions in the time domain, with a rectangular box-shaped Fourier transform in the frequency domain. 
Another approach to decompose a real-valued signal into the superposition of complexvalued wavelets $\psi_{\{m, n\}, k}(t)$ having complex-valued combination coefficients $a_{\{m, n\}, k}$ consists in the use of the circular wavelets. The whole set of band circular wavelets is generated by:

$$
\psi_{\{m, n\}, k}(t)=\frac{1}{n-m} \sum_{j=m}^{n-1} \exp \left[\mathrm{i} 2 \pi j\left(t-\tau_{\{m, n\}, k}\right)\right]
$$

where the notation $\{m, n\}$ is used to denote a wavelet occupyng the band of circular frequencies from $2 \pi m$ to $2 \pi n$, with $n>m ; \tau_{\{m, n\}, k}=k /(n-m)$ is a deterministic time shift of the wavelets belonging to the $\{m, n\}$-indexed frequency band; $k=0, \ldots,(n-m-1)$ is an integer number; and $\mathrm{i}=\sqrt{-1}$ is the imaginary unit.

In the discrete wavelets transform, the complex-valued combination coefficients $a_{\{m, n\}, k}$ of a target signal in the time domain are calculated by a discrete convolution of the signal, say $\ddot{U}_{\mathrm{g}}(t)$, with the band wavelets $\psi_{\{m, n\}, k}(t)[3]$ :

$$
a_{\{m, n\}, k}=\sum_{\ell=0}^{N} \ddot{U}_{\mathrm{g}}\left(\frac{t_{\ell}}{t_{f}}\right) \cdot \frac{\Delta t}{t_{f}} \cdot \psi_{\{m, n\}, k}\left(t_{\ell}\right)
$$

where $t_{\ell}=\ell \cdot \Delta t$ is the $\ell$ th of the $N=t_{f} / \Delta t$ discrete time instants at which the signal is discretized, being $\Delta t$ and $t_{f}$ the sampling interval and the time duration of the selected signal, respectively.

In this paper, the circular wavelets have been used for the randomisation of the target signal $\ddot{U}_{\mathrm{g}}(t)$. Specifically, the generation of the $r$ th fully non-stationary artificial sample of the random process can be evaluated by the following formula:

$$
f^{(r)}(t)=2 \sum_{\{m, n\}} \sum_{k=0}^{n-m-1} \sum_{j=m}^{n-1} \frac{\left|a_{\{m, n\}, k}\right|}{n-m} \times \cos \left[\mathrm{i} 2 \pi j\left(t-\tau_{\{m, n\}, k}\right)+\theta_{\{m, n\}, k}+\phi_{\{m, n\}, k}^{(r)}\right]
$$

where $\phi_{\{m, n\}, k}^{(r)}$ is the $r$ th realization of a random variable uniformly distributed over the interval $[0,2 \pi]$ while $\bar{\theta}_{\{m, n\}, k}=\arg \left\{\bar{a}_{\{m, n\}, k}\right\}$ is the corresponding deterministic phase of the complex-valued coefficient of the target signal.

\section{EVOLUTIONARY POWER SPECTRAL DENSITY FUNCTION METHOD FOR MODELLING SEISMIC ACTION}

For the sake of completeness, the four-step method recently proposed in [12] for generating random samples of a fully non-stationary zero-mean Gaussian process consistent with a target accelerogram is summarized in this section.

First, the time axis is divided in $n$ contiguous time intervals, in which a uniformly modulated process is introduced as the product of a deterministic modulating function, $a(t)$, times a stationary zero-mean Gaussian sub-process $X_{k}(t)$, whose power spectral density $(P S D)$ function $G_{X_{k}}(\omega)$ is filtered by two Butterworth filters: 


$$
G_{X_{k}}(\omega)=\beta_{k}\left(\frac{\omega^{2}}{\omega^{2}+\omega_{H, k}^{2}}\right)\left(\frac{\omega_{L, k}^{4}}{\omega^{4}+\omega_{L, k}^{4}}\right) \frac{\rho_{k}}{\pi}\left(\frac{1}{\rho_{k}^{2}+\left(\omega+\Omega_{k}\right)^{2}}+\frac{1}{\rho_{k}^{2}+\left(\omega-\Omega_{k}\right)^{2}}\right) ; k=1, \ldots, n
$$

where $\beta_{k}$ is evaluated in such a way that the sub-process $X_{k}(t)$ possesses unit variance. The predominant circular frequency $\Omega_{k}$ and the frequency bandwidth $\rho_{k}$ in Eq. (4) depend on the occurrences of maxima $P_{k}$ and of zero-level up-crossings $N_{0, k}^{+}$of the target accelerogram, in the various $k$ intervals:

$$
\Omega_{k} \cong \frac{2 \pi N_{0, k}^{+}}{\Delta T_{k}} ; \quad \rho_{k} \simeq \frac{\pi N_{0, k}^{+}}{2 \Delta T_{k}}\left[\pi-2 \frac{N_{0, k}^{+}}{P_{k}}\right]
$$

Second, the modulating function $a(t)$ is evaluated by least-square fitting the cumulative expected energy function of the stochastic process to the cumulative energy function $E_{\ddot{U}_{g}}(t)$ of the target accelerogram subdivided in three-time intervals:

$$
a(t)=\sum_{j=1}^{2} \bar{a}_{j}(t) \mathbb{W}\left(t_{j-1}, t_{j}\right)+a\left(t_{2}\right) \exp \left[\frac{t-t_{2}}{t_{f}-t_{2}} \ln \left(\frac{\left|\ddot{U}_{\mathrm{g}}\left(t_{f}\right)\right|}{a\left(t_{2}\right)}\right)\right] \mathbb{W}\left(t_{2}, t_{3}\right) .
$$

in which $\mathbb{W}\left(t_{j-1}, t_{j}\right)=\mathbb{U}\left(t-t_{j}\right)-\mathbb{U}\left(t-t_{j-1}\right)$ is the window function and $\mathbb{U}(t)$ the Heaviside unit step function.

Third, the $i$ th sample of the random process is generated via the superposition of harmonic functions with random phases:

$$
F_{0}^{(i)}(t)=a(t) \sqrt{2 \Delta \omega}\left[\sum_{k=1}^{n} \sum_{r=1}^{m_{N}} \mathbb{W}\left(t_{k-1}, t_{k}\right) \sin \left(r \Delta \omega t+\theta_{r}^{(i)}\right) \sqrt{G_{X_{k}}(r \Delta \omega)}\right]
$$

being $\theta_{r}^{(i)}$ the random phase angles, uniformly distributed over the interval $[0,2 \pi]$ and $m_{N}$ the number of parts in which the $k$ th PSD function $G_{X_{k}}(\omega)$ is discretized with a $\Delta \omega$ frequency sampling interval.

Finally, the spectrum-compatibility is obtained by reducing the gap between the mean spectrum of the generated samples $\bar{S}^{(j-1)}\left(\omega, \zeta_{0}\right)$ and the target one $S^{(T)}\left(\omega, \zeta_{0}\right)$, through the introduction of a corrective iterative PSD function $\bar{G}_{X_{k}}^{(j)}(\omega)$ :

$$
\bar{G}_{X_{k}}^{(j)}(\omega)=\bar{G}_{X_{k}}^{(j-1)}(\omega) \frac{S^{(T)}\left(\omega, \zeta_{0}\right)^{2}}{\bar{S}^{(j-1)}\left(\omega, \zeta_{0}\right)^{2}}
$$

being $\bar{G}_{X_{k}}^{(0)}(\omega)=1[14]$ and $\zeta_{0}$ the viscous damping.

According to the formulation described in [15], the generic spectrum-compatible sample can be generated as:

$$
\bar{F}_{0}^{(i)}(t)=a(t) \sqrt{2 \Delta \omega}\left[\sum_{k=1}^{n} \sum_{r=1}^{m_{N}} \mathbb{W}\left(t_{k-1}, t_{k}\right) \sin \left(r \Delta \omega t+\theta_{r}^{(i)}\right) \sqrt{\bar{G}_{X_{k}}^{(j)}(r \Delta \omega) G_{X_{k}}(r \Delta \omega)}\right] .
$$


Different types of spectrum-compatibility can be achieved, modifying the corrective iterative $P S D$ function term $\bar{G}_{X_{k}}^{(j)}(\omega)$ as shown in [16].

\section{NUMERICAL APPLICATION}

In order to quantify the influence of the way of modelling the expected seismic action, two set of one hundred Fully Non-Stationary samples consistent with a target seismic accelerogram have been generated using the proposed wavelet-based method (CWT) and the evolutionary piecewise power spectral density (EPSD) function procedure proposed in [12].

A comparison in terms of displacement response spectra, computed for SDoF oscillators with viscoelastic damping based on the Maxwell model is also presented in the paper.

\subsection{Target Motion}

The North-South component of the ground motion recorded at Vasquez Rocks Park during the 1994 Northridge earthquake has been used in the following as target accelerogram $\ddot{U}_{\mathrm{g}}(t)$.

The selected ground motion, downloaded from the Peer database [17], having a moment magnitude $M_{\mathrm{w}}=6.7$ and a site-source distance $R_{\mathrm{JB}}=23.1 \mathrm{~km}$ [18], has been recorded with a sampling time $\Delta t=0.02 \mathrm{~s}$ by a station having an average shear wave velocity in the upper $30 \mathrm{~m}$ equal to $V_{s, 30}=996 \mathrm{~m} / \mathrm{s}$ (EC8 [19], soil class "A"). The total Intensity of the target accelerogram, having an overall duration $t_{f}=36.6 \mathrm{~s}$, is equal to $I_{0}=1.9 \mathrm{~m}^{2} / \mathrm{s}^{3}$, while the total number of zero-level up-crossings and peaks are $N_{0}{ }^{+}=196$ and $P_{0}=212$, respectively.

In Figure 1, the trend of the time-history of the analysed target accelerogram is reported.

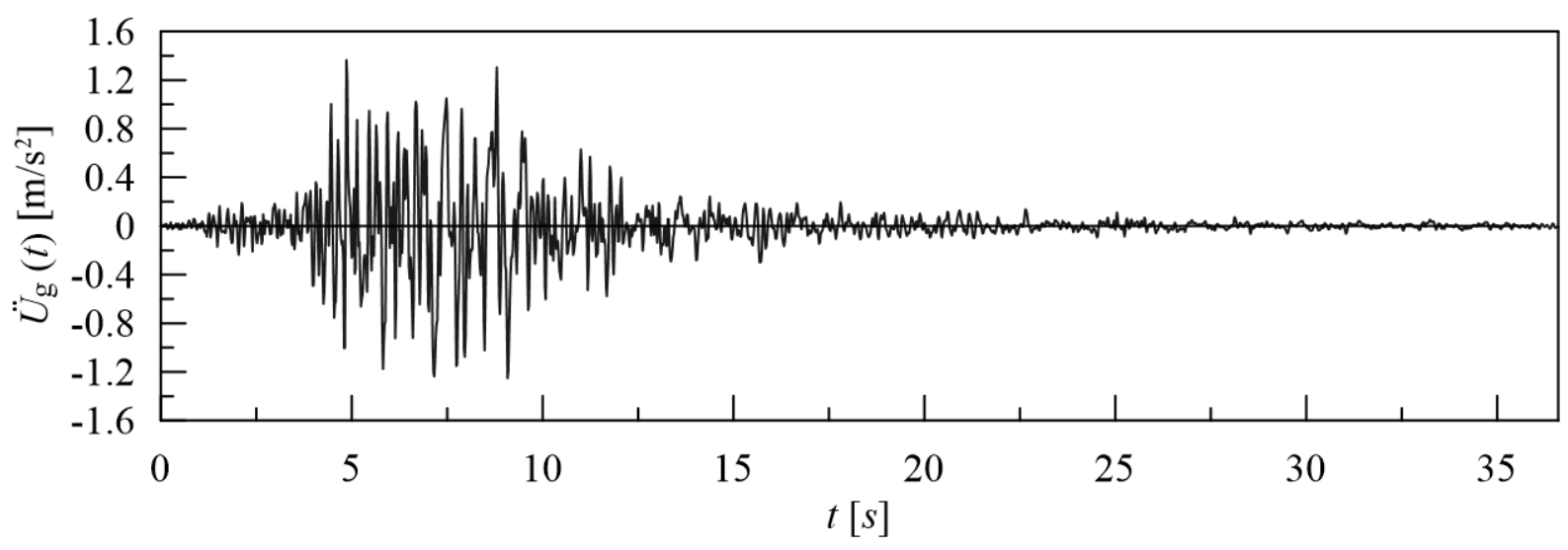

Figure 1: Time histories of the 1994 Northridge earthquake accelerogram.

\subsection{Wavelet-based method}

A fundamental step of the proposed Circular Wavelet Transform (CWT) method consists in the correct choice of the amplitudes of bands in which to divide the frequency domain of the selected signal $\ddot{U}_{\mathrm{g}}(t)$.

In this paper, three different schemes have been investigated considering a different subdivision of the cumulative Fourier energy function evaluated as:

$$
E(f)=\int_{0}^{f c}\left(\left|\mathscr{F}\left[\ddot{U}_{\mathrm{g}}(t)\right]\right|\right)^{2} \mathrm{~d} f
$$

$f c$ being the cut-off frequency and $\mathscr{F}\left[\ddot{U}_{\mathrm{g}}(t)\right]$ the Fourier transform of the target signal. 
Accordingly, the frequency domain has been subdivided in frequency bands of:

1) equal-spaced bandwidths $(E S B)$;

2) constant energy bandwidths $(C E B)$;

3) non-uniform energy bandwidths (NUEB).

In the latter case, the choice of the bandwidths has been made according to the main changes in the slope of the $E(f)$ function. In all three investigated schemes a subdivision into 10 parts has been carried out.

The representation of the subdivision of the cumulative Fourier energy function for the three analysed schemes is reported in Figure 2.

In Figure 3, the mean values of the modules of the Fourier spectra, obtained for the three analysed configurations, are compared with the target one. It can be observed that the mean value of the module of the Fourier Spectrum of the generated samples is in a good agreement with the target one only in the case of subdivision of the frequency domain with energy criterium (schemes 2 and 3 ).

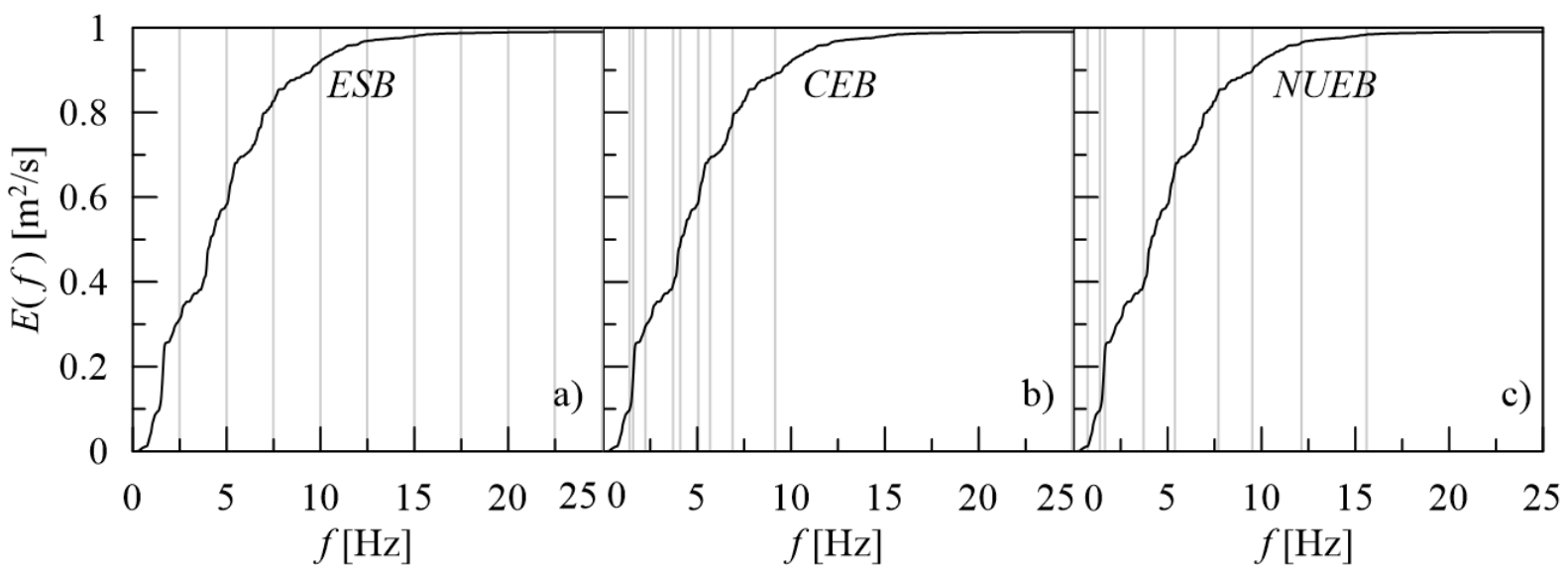

Figure 2: Representation of cumulative Fourier energy function (black line) together with the frequency bands subdivision (grey vertical lines) for the three investigated schemes: a) equal-spaced bandwidths (ESB); b) constant energy bandwidths $(C E B)$; c) non-uniform energy bandwidths $(N U E B)$.

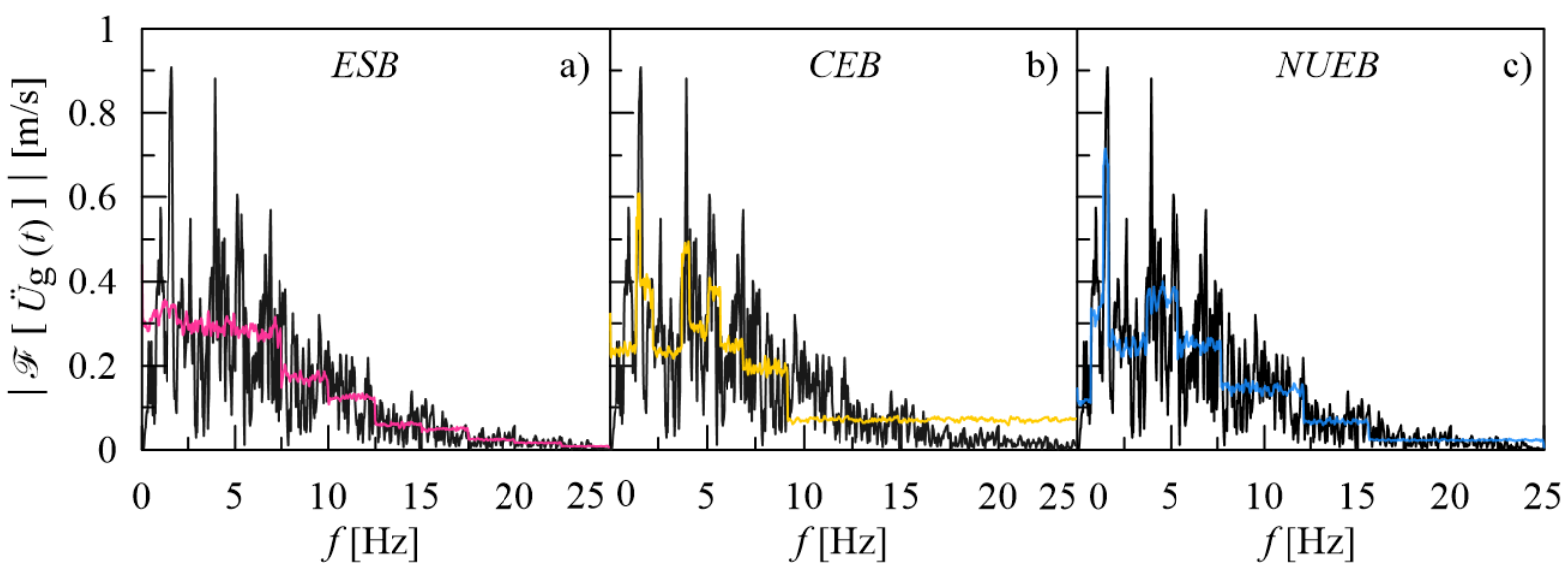

Figure 3: Comparison among mean Fourier spectrum module of the generated samples by the $C W T$ method and the target one (black line) considering: a) equal-spaced bandwidths (ESB); b) constant energy bandwidths $(C E B)$; c) non-uniform energy bandwidths (NUEB). 
In Figure 4, three generic samples (coloured lines) are plotted against the target accelerogram (black line), showing that in all cases the variation in amplitude appears to be preserved in the time domain.

In Figure 5, the acceleration spectrum of the target accelerogram (red line) is compared to that obtained as the mean value of 100 samples (black solid line). The confidence interval evaluated as the mean value plus/minus the corresponding standard deviation (black dotted lines) and the envelope of the maximum and minimum values of all samples (shaded area) are also reported in Figure 5.

In Figures $6 \mathrm{a}$ and $6 \mathrm{~b}$, further comparisons are offered in terms of the cumulative energy functions $I_{0}(t)$ and the cumulative zero-level up crossing functions $N_{0}^{+}(t)$, respectively.

From the observation of the results obtained in the time and frequency domain, it emerges that a subdivision of the frequency domain in non-uniform energy bandwidths (third scheme) leads to outcomes statistically closer to those of the target event.

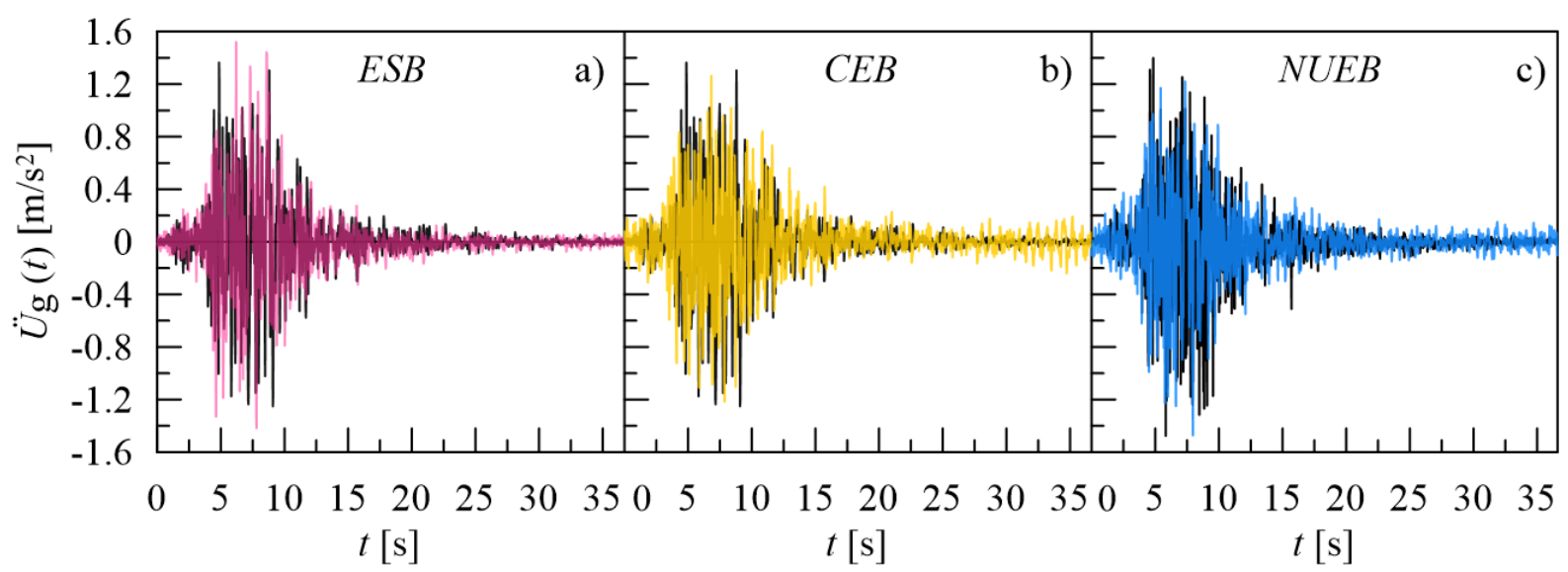

Figure 4: Comparison among the target accelerogram (black line) and the $i$ th generated sample by the proposed CWT method, considering a subdivision of the frequency domain in: a) equal-spaced bandwidths (magenta line);

b) constant energy bandwidths (yellow line); c) non-uniform energy bandwidths (blue line).

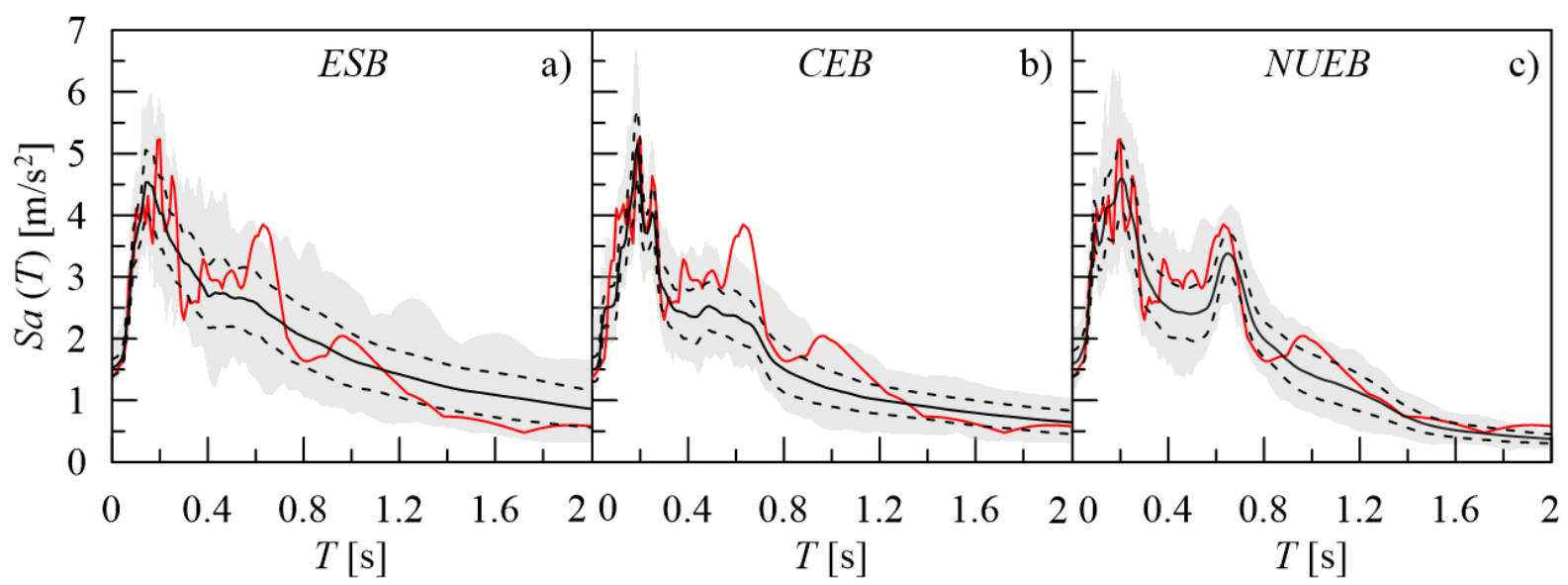

Figure 5: Comparison among the acceleration response spectrum of the target accelerogram (red solid line) with statistics of the artificial ones: mean value function (black line); mean value plus/minus standard deviation functions (black dashed lines); envelope of the maximum and minimum values of all samples (shaded area); consid-

ering a subdivision of the frequency domain in: a) equal-spaced bandwidths $(E S B)$; b) constant energy bandwidths $(C E B) ; \mathrm{c})$ non-uniform energy bandwidths $(N U E B)$. 

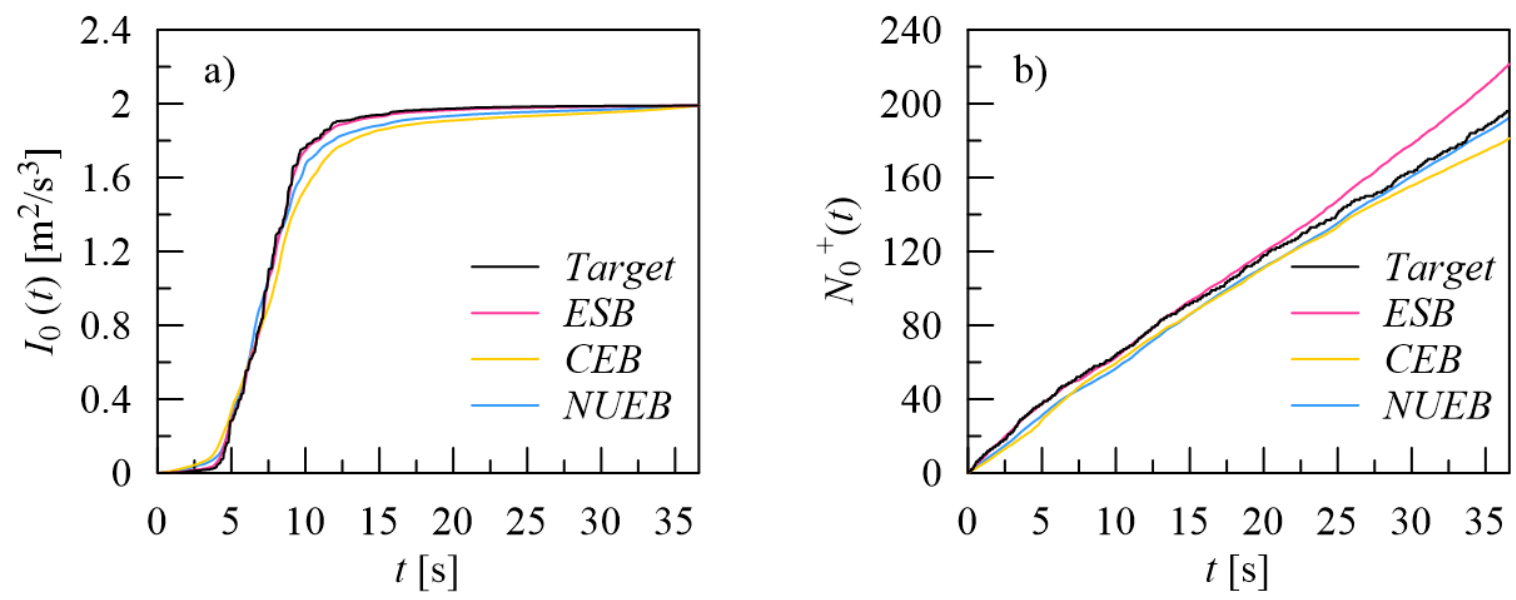

Figure 6: Comparison between the target (black line) and the averages a) cumulative energy functions, b) zero level up crossing functions, considering: equal-spaced bandwidths ( $E S B)$; constant energy bandwidths $(C E B)$; c) non-uniform energy bandwidths (NUEB).

\subsection{Evolutionary Power spectral density function (EPSD) method}

Using the iterative procedure described in Section 3, through 4 iterations, a set of 100 artificial accelerograms has been generated using appropriate modulating and PSD functions which allowed to preserve the amplitude and the frequency content of the target ground motion. Further details about the parameters that characterize these functions can be found in [15].

\subsection{Dynamic analyses of linear visco-elastic systems}

In this section, displacement response spectra have been computed to illustrate the structural response of SDoF oscillators provided with linear viscoelastic damping ruled by:

$$
m \ddot{u}(t)+r(t)=\ddot{U}_{\mathrm{g}}(t)
$$

where $m$ is the mass of the system, $\ddot{u}(t)$ is the second-order derivative of the displacement relative to the ground $u(t)$ and $r(t)$ is the reaction force that in the Maxwell Model is given by:

$$
r(t)=k_{0} u(t)+k_{M} \lambda(t)
$$

being $k_{0}$ the equilibrium modulus and $\lambda(t)$ the additional internal variable taken as the deformation of the Maxwell element, ruled by the state equation:

$$
\dot{\lambda}(t)=\dot{u}(t)-\frac{\lambda}{\tau}
$$

where:

$\tau=\frac{c_{M}}{k_{M}}$

$k_{M}$ and $c_{M}$ being the elastic stiffness and the viscous coefficient of the Maxwell element, re-

spectively, while $u(t)$ is the first-order derivative of the displacement $u(t)$.

In this paper the values of $k_{M}=400 \mathrm{~N} / \mathrm{m}$ and $c_{M}=40 \mathrm{~N} \mathrm{~s} / \mathrm{m}$ have been assumed, according to case examined in [20]. 
In Figure 7, the displacement spectra of the target accelerogram (red lines) are compared to that obtained as the mean value of 100 samples (black continues lines), using: a) the Circular Wavelet transform method, with a subdivision of the frequency domains in non-uniform energy bandwidths (case 3); b) the Evolutionary Power spectral density function method.

The confidence intervals are evaluated as the mean values plus/minus the corresponding standard deviations (black dotted lines); the envelope of the maximum and minimum values of all samples (shaded area) are also reported in Figure 7.
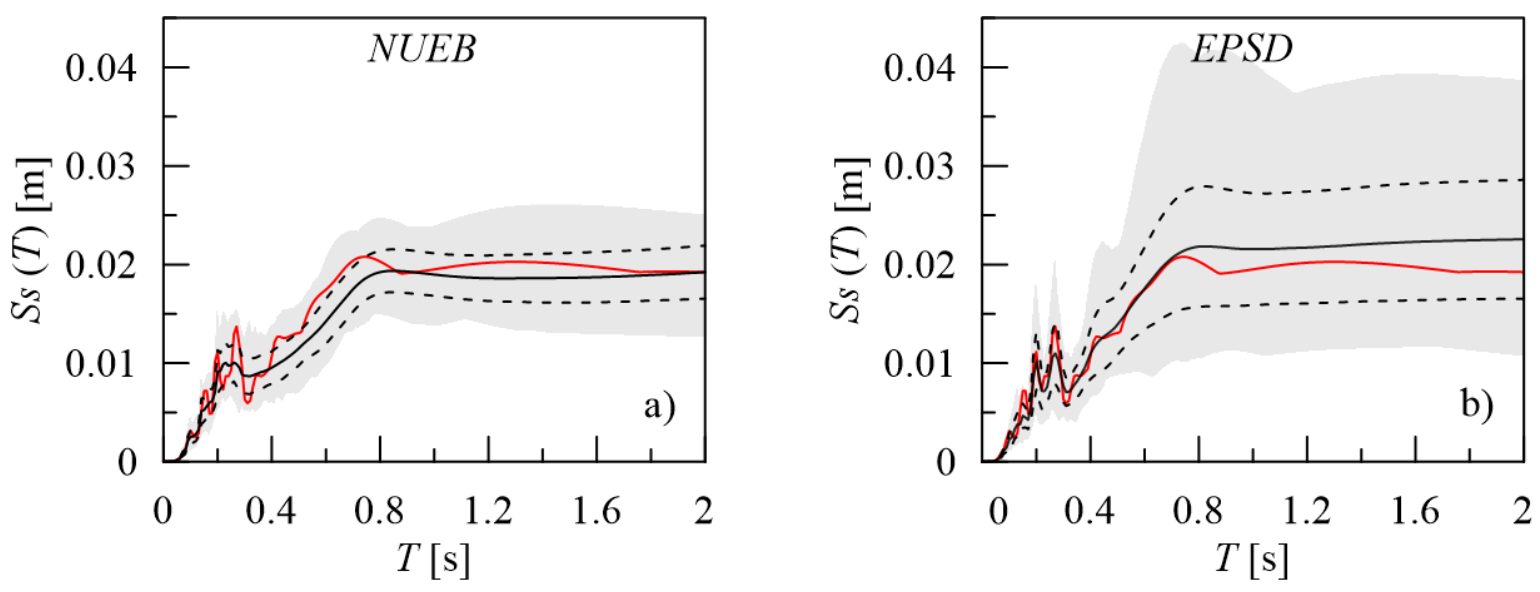

Figure 7: Comparison between target displacement spectrum (red line) of a system with linear viscoelastic damping based on the Maxwell model together with statistics of the artificial ones: mean value function (black solid line); mean value plus/minus standard deviation functions (black dashed lines); envelope of the maximum

and minimum values of all samples (shaded area): a) $C W T$ method with non-uniform energy bandwidths

$(N U E B)$, b) EPSD function method.

\section{CONCLUSIONS}

Seismic response of structural and geotechnical systems is often highly sensitive to the way of modelling the dynamic action. In order to verify the influence of different fully non-stationary artificial accelerograms generation procedures, two stochastic methods, have been compared.

The $C W T$-method, proposed in this paper, consists in a phase angle rotation of the circular wavelets in the complex-valued space and allows the generation of the required number of fully non-stationary samples without the need of defying the evolutionary power spectral density function of the ground acceleration.

The choice of the number and the amplitude of bands in which to divide the frequency domain is an important step to generate samples with the desired time-variation of amplitude and frequency content.

The numerical results show that the generated samples obtained by the $C W T$ method are close to the target one in the case of the subdivision of the frequency domain in non-uniform energy bandwidths; furthermore, the average displacement spectrum of the artificial samples generated by the $C W T$ method and computed for SDoF oscillators with viscoelastic damping has a trend close to the target one.

The application of iterative corrections in the EPSD method allows the target displacement spectrum to completely fall into the confidence interval evaluated as the mean value plus/minus standard deviation of the displacement spectrum of the generated samples. It appears that the EPSD function method allows to obtain samples having a displacement response spectrum closer to the target one; however, it tends to be more complex and requires several iterative steps. 


\section{REFERENCES}

[1] F. Genovese, D. Aliberti, G. Biondi, E. Cascone, A procedure for the selection of input ground motion for 1D seismic response analysis. Earthquake Geotechnical Engineering for Protection and Development of Environment and construction. F. Silvestri and N. Moraci eds. 7th International Conference on Earthquake Geotechnical Engineering (ICEGE 2019), Rome, Italy, June 17-20, 2019, pp. 2591-2598, 2019.

[2] J.J. Bommer, B. Acevedo, The use of real earthquake accelerograms as input to dynamic analysis. Journal of Earthquake Engineering, 8, Special issue, 1, pp. 43-91, 2004.

[3] D. Cecini, A. Palmeri, Spectrum-compatible accelerograms with harmonic wavelets, Computers and Structures, 147, pp.26-35, 2015.

[4] M. Rota, E. Zuccolo, L. Taverna, M. Corigliano, C. G. Lai, A. Penna, Mesozonation of the Italian territory for the definition of real spectrum-compatible accelerograms, Bulletin of Earthquake Engineering, 10, pp. 1357-1375, 2012.

[5] F. Genovese, D. Aliberti, G. Biondi, E. Cascone, Influence of Soil Heterogeneity on the Selection of Input Motion for 1D Seismic Response Analysis. F. Calvetti, F. Cotecchia, A. Galli, C. Jommi eds, Geotechnical Research for Land Protection and Development. CNRIG 2019, Lecture Notes in Civil Engineering, Springer, Cham, 40, pp. 694-704, 2020.

[6] F. Genovese, D. Aliberti, G. Biondi, E. Cascone. Geotechnical aspects affecting the selection of input motion for seismic site response analysis. M. Papadrakakis, M. Fragiadakis eds, $7^{\text {th }}$ Computational Methods in Structural Dynamics and Earthquake Engineering (COMPDYN 2019), 24-26 June 2019, Crete, Greece, 1, pp. 151-161, 2019.

[7] F. Genovese, Influence of Soil Non-linear Behaviour on the Selection of Input Motion for Dynamic Geotechnical Analysis. M. Barla, A. Di Donna, D. Sterpi D. eds, Challenges and Innovations in Geomechanics, $16^{\text {th }}$ International Conference of the International Association for Computer Methods and Advances in Geomechanics (IACMAG 2021), Lecture Notes in Civil Engineering, Springer, Cham, 126, pp. 588-596, 2021.

[8] G. Stefanou, S. Tsiliopoulos, Estimation of evolutionary power spectra of seismic accelerograms. M. Papadrakakis, M. Fragiadakis (Eds), $7^{\text {th }}$ International Conference on Computational Methods in Structural Dynamics and Earthquake Engineering, 3, pp. 5880-5888, 2019.

[9] P. Cacciola, A stochastic approach for generating spectrum-compatible fully nonstationary earthquakes, Computers and Structures, 88, pp. 889-901, 2010.

[10] J. Iyama, H. Kuwamura, Application of wavelets to analysis and simulation of earthquake motions, Earthquake Engineering and Structural Dynamics, 28, no. 3, pp. 255272, 1999.

[11] A. Giaralis and P. D. Spanos, Wavelet-based response spectrum compatible synthesis of accelerograms - Eurocode application (EC8), Soil Dynamics and Earthquake Engineering, 29, no. 1, pp. 219-235, 2009.

[12] G. Muscolino, F. Genovese, G. Biondi, E. Cascone, Generation of Fully Non-Stationary Random Processes Consistent with Target Seismic Accelerograms, Soil Dynamics and Earthquake Engineering, 141(106467), pp. 1-14, 2021. 
[13] D. Newland, Harmonic and musical wavelet. Proceedings, Royal Society A, 44, pp.605620, 1994.

[14] E.H. Vanmarcke, D.A. Gasparini, Simulated earthquake ground motions. K - Seismic Response Analysis of Nuclear Power Plant Systems K1 - Ground Motion and Design Criteria SMiRT 4, San Francisco, USA, 1977.

[15] F. Genovese, G. Muscolino, G. Biondi and E. Cascone. Generation of artificial accelerograms consistent with earthquake-induced ground motions. M. Papadrakakis, M. Fragiadakis, C. Papadimitriou eds, $11^{\text {th }}$ International Conference on Structural Dynamic, (EURODYN 2020), Virtual, Athens, Greece, November 23-26 2020; Code 165382, 2 , pp. 3027-3042, 2020.

[16] F. Genovese, G. Muscolino, G. Biondi, E. Cascone. A novel method for the generation of fully non-stationary spectrum compatible artificial accelerograms. M. Papadrakakis, M. Fragiadakis eds, $8^{\text {th }}$ Computational Methods in Structural Dynamics and Earthquake Engineering, (COMPDYN 2021), Virtual, Athens, Greece, June 27-30 2021.

[17] Ancheta, Robert B. Darragh, Jonathan P. Stewart, Emel Seyhan, Walter J. Silva, Brian S.J. Chiou, Katie E. Wooddell, Robert W. Graves, Albert R. Kottke, David M. Boore, Tadahiro Kishida, and Jennifer L. Donahue, PEER NGA-West2 database. Pacific Earthquake Engineering Research Center/03; California; 2013.

[18] W.B. Joyner, D.M. Boore, Peak horizontal acceleration and velocity from strong motion records including records from the 1979 Imperial Valley, California, earthquake. Bulletin of the Seismological Society of America; 71, pp. 2011-2038, 1981.

[19] EC8 (European Committee for Standardization, Eurocode 8). Design of structures for earthquakes resistance-Part 1: General rules, seismic actions and rules for buildings, (EN 1998-1).

[20] G. Muscolino, A. Palmeri, Dynamic analysis of viscoelastically damped structures, B.H.V. Topping, G. Montenero, R. Montenegro eds, $8^{\text {th }}$ International conference on computational structures technology, Las Palmas de Gran Canaria-Spain, pp. 325-347, 2006. 